1 c.cm. capacity, and two needles are used, a long and a short; the needles have tapering barrels extending the greater portion of their length to give them increased rigidity.

One syringeful is injected with a long needle in the upper and posterior parts of the septum under the mucoperiosteum covering the bone, in the neighbourhood of the naso-palatine nerve. This serves to anaesthetize the posterior part of the septum. The second syringeful is entered in the anterior and upper part of the septumthat is, in the neighbourhood of the internal twig of the nasal branch of the ophthalmic nerve. A third syringeful is divided between the line of incision and the septum near the floor, especially where the anterior: palatine canal emerges. The same amount is injected on the other side. The mouth is then covered with a mask of gauze, held in situ with adhesive strapping, and the operation proceeded with. After the first syringeful is injected, certain circulatory disturbances in varying degrees are noticed, the patient usually becomes blanched, beads of perspiration exude, the heart thumps distressingly, and oscasionally, in fatty subjects and persons addicted to much smoking, it beats quite irregularly, intermitting one now and again. Others complain of intense pain in the lower part of the back and abdomen-I am inclined to believe this is due to great and tense pulsation of the abdominal aorta. A good many have headaches which last during the operation or supervene some hours later. All these effects are attributable to the action of adrenalin in raising the peripheral pressure and stimulating the heart. These symptoms shortly subside, and the injection may be resumed. The increased pulsation generally occurs in diminishing degree after each injection; sometimes from fifteen to twenty minutes afterwards there is a reactionary fall of blood pressure, and the patient partially collapses, due possibly to the late absorption of cocaine. I have, however, never seen this phenomenon interrupt the course of the operation since I have placed them supine on the table.

With local anaesthesia properly done one can gajn complete insensibility to operative manipulations, but there is always a distressing noise transmitted in a magnified degree to the ears when the bone of the superior maxillary crest and vomer are punched out or chiselled away. It is on this account that one is induced to turn to a combination of local anaesthesia and narcosis in nervous and timid patients. With a combination of general and local anaesthesia the patients are unconscious of the circulatory disturbances, grating of the bone, headache, and manipulation of the nose so distasteful to females. The element of danger is increased, however, as some reported fatalities show, but with a competent anaesthetist, and attentive observation of the effect of the injections, the risk is reduced to a minimum.

With regard to the method in detail, anaesthesia is induced with a mixture of chloroform and ether (in the proportion of 1 to 2 ) ; the induction should proceed slowly, and when the patient is barely unconscious, that is, when the breathing is automatic but the reflexes brisk, half a syringeful of the $\frac{1}{5}$ per cent. cocaine and normal saline above mentioned, to which is added two drops of adrenalin chloride $(1$ in 1,000$)$ to the cubic centimetre, is injected in the posterior part of the septum. The effect should be watched, and if increased pulsation ensues wait until it subsides a fittle before injecting any more. The same sites of injection are selected as in the description given of local anaesthesia. I always regard increased pulsation of the heart-beat and a strong pulse as safe signs. Great pallor and no increase in the pulse tension or strength of the heart's beat should excite suspicion, and the injection must be snspended, the operation being pursued without further infiltration. Irregularity of the beat occurs occasionally, although the heart may beat strongly. 'This I regard as no deterrent, but intermittent beating associated with a pulse of a weak and feeble character is distinctly.interdictory. In this connexion I should like to interpolate a report of 3 cases showing the alarming effect of the sudden absorption of adrenalin on the system-an effect due, in my opinion, to the action of adrenalin on a circulatory system depressed by chloroform, and not the result of a nasal reflex, for the action only supervened after a latent period corresponding to the approximate time of absorption.

\section{CA8E I.}

A pale and anaemic woman with a feeble circulation was fairly deeply anaesthetized before the submucous injection was thrown in. About a minute after this the patient suddenly became intensely pallid, respiration ceased, and the pupils suddenly dilated. She was promptly inverted, and after about twenty to thirty seconds of anxious waiting she gave a sighing Artificial respiration was not resorted to.

\section{CASE II.}

This case occurred in a woman about a month later almost in the same way, except that the untoward symptoms appeared earlier. Inversion restored her breathing, and the operation was completed.

In both these cases the effect was at first attributed to chloroform overdose, but in the light of a further case which occurred a week later at the Ear and Throat Hospital this opinion was modified.

CASE III.

This was a case of endo-nasal operation for antral suppuration. The patient was rather deeply anaesthetized, and I injected $\frac{1}{3}$ c.cm. of normal saline with a few drops of adrenalin ( 1 in 1,000) added into the anterior end of the inferior turbinate, with the object of resecting this bloodlessly. Within ten seconds respiration ceased, the pulse was suppressed, and the pupils widely dilated. She was inverted immediately, and respiration gradually restored, the pulse becoming stronger by slow degrees.

The effect was attributable to adrenalin as no cocaine was present in the solution. It occurred earlier in this case owing to the wide venous space into which it was injected and more rapid absorption into the system. One can only conjecture how it acts. Is it by causing anaemia of the bulb, or may there be some slight toxic action on the respiratory centre? Does the heart first fail owing to spasm of the coronary arteries? The whole condition supervenes so rapidly it makes accurate observation difficult. After the injection the mouth, chin, and lips are well vaselined over; a piece of gauze fourfold thick and about 3 in. wide is doubled across a strip of adhesive strapping, this mask is laid across the mouth and affixed by the strapping to the cheeks. The mouth is kept half open by a Doyen's gag, and the anaesthetic administered by dropping it on the gauze, which is kept slightly raised from contact with the mouth by the hand. Very little anaesthetic is required, as the local infiltration numbs all pain and consciousness need only be barely abolished.

\section{Conclusions.}

For local anaesthesia (1) all healthy individuals who do not mind slight discomfort; adipase subjects if they will submit to it.

For combination of general and local-nervous and timid subjects who prefer abolition of consciousness in whom the heart is quite sound; (2) children if operation is really necessary. Great care should be exercised in anaemic pallid subjects with a feeble pulse. Over a hundred cases have been done with a combination of local and general anaesthesia, and more than five hundred with local anaesthesia, with no fatalities.

A preliminary injection of scopolamine and morphine is to be deprecated as the morphine seems to inhibit the ischaemic effect of the adrenalin.

\section{ATROPINE AND OPEN ETHER ADMINISTRATION}

BY H. BELLAMY GARDNER, M.R.C.S., L.R.C.P.LOND.,

FORMERLY ANAFSTHETIST AND INSTRUCTOR IN ANAESTHETICS AT CHARING CROSS HOSPITAL.

Is order to facilitate the general adoption of the open method of ether administration I venture to place on record some modifications of technique which contribute towards the ease of the induction and the perfection of the resulting anaesthesia.

An initial eommunication on this subject appeared in the British Medicat Journal for November 23rd, 1907, and a description of the method used in the JouRNaL for January 18th, 1908. A paper was also read at the annual meeting of the British Medical Assoeiation in London, 1910, and reported in the JouRNAL for September 17th, 1910. 
The Technique.

1. One hour before the administration a hypodermic injection of atropine sulphate $Y_{1}^{1} \sigma$ grain is given with the object of preventing the considerable secretion of mucus in the air passages which may otherwise be caused by ether. This also prevents sweating during the operation, and thereby retains the body heat. Atropine is also a respiratory stimulant and a vagal obtundent, both valuable qualities unhampered by contraindications. Morphine is not advantageous for routine employment, because it appears to induce undue oozing of blood from the wound during operation, and certainly retards the reappearance of the laryngeal reflex and the resumption of consciousness during recovery.

2. When the patient is in position for the ether administration a small mouth prop is inserted between the side teeth and held in position by light pressure upwards upon the lower jaw with the administrator's left hand.

3. A ring pad made of gauze is then rested on the patient's face encircling the nose and mouth.

4. The wire mask fitted with a pad made of sixteen layers of gauze is now laid upon the ring pad, and the patient instructed to breathe in and out slowly through the mouth.

5. The administrator now talks quietly to the patient all the time, while he drops ether continuously upon the mask until unconsciousness supervenes.

6. The mouth is then opened wider with a jaw gag, a tongue clip with finger loop is inserted, and the tongue drawn slightly forward away from the pharynx.

The mouth prop is then readjusted, leaving the mouth partly open as at first. The ring pad and mask are then replaced and the ether drops continued.*

By means of this procedure all further anxiety as to the patency of the airways is removed, and laboured respiration entirely avoided.

\section{Practical Advantages.}

These may be shortly summed up under the following headings :

1. A smooth induction period of six to eight minutes,

2. Very early clouding of the mental faculties.

3. Tranquil regular respiration with abdominal relaxation.

4. Absence of mucus in the air passages.

5. No unusual oozing of blood from the wound.

6. Extraordinary safety, alterations in the patient's position or disturbances of vital structures producing barely any depression.

7. Maintenance of normal blood pressure and absence of shock owing to the obtundent effects of both atropine and ether.

8. Suitability for goître operations and adenoid and tonsil extirpation.

9. The patient may be safely propped upright on pillows on return to bed.

10. No after-effects, vomiting being quite rare.

11. Acidosis improbable.

12. Unusual value therefore in septic conditions and those with flagging circulation.

After four years' employment of open ether administration, in contrast with thirteen years' previous use of other methods, I cannot refrain from strongly urging its general acceptance as a substitute for chloroform and mixtures in all routine surgical work on account of its extreme simplicity, safety, and convenience for the operator, administrator, and patient.

* The mouth props, tongue clip, mask, and sewn gauze pads may be obtained from Hessrs. Allen and Hanburys of Wigmore Street.

THE Lord Mayor, who presided at the annual meeting of the St. Mark's Hospital for Cancer, Fistula, and other Diseases of Rectum, London, E.C., on February 16th, said that his knowledge of the institution was of long standing, since more than fifty years ago he had the pleasure of knowing the founder, and had assisted the staff of the hospital at operations in the early days on a number of occasions. The annual report, moved by Dr. A. W. Oxford, showed that 643 in-patients and 1,997 outpatients had been under treatment during 1911. The addition of six beds increased the cost of provisions and a ialling-off in the income had produced a deficit of $\$ 621$. A vote of thanks to the honorary medical staff, moved by the Lord Mayor, was acknowleged by Mr. Swinford Edwards.

\section{LEFT-SIDED SUBPHRENIC ABSCESS DUE TO PERFORATED DUODENAL ULCER.}

\author{
BY
}

H. D. ROLLESTON, M.D., F.R.C.P., sENIOR PHYSICIAN, ST. GEORGE's HOSPITAL; PHYSICIAN, VICTORIA
HOSPITAL FOR CHILDREN.

This case is remarkable from two points of view, the clinical and the anatomical.

A man aged 66 was admitted on March 1st, 1910, to St. George's Hospital with severe abdominal pain, worse on the right side, and extending from the right costal margin to the iliac fossa. The right half of the abdomen was tender, rigid, distended, and resonant; the liver dullness was diminished. There were signs of a small pleural effusion on the right side. The tongue was dry and coated, and the patient looked pale, ill, and dired and distressed. The temperature was $99^{\circ}$ on admission, the pulse 88 , and the respirations 24 . The urine, 1030, acid, contained indican, gave a slight reduction with Fehling's solution and was free from albumen and pus. He had not been sick, and was not so at any time after admi

Past History.-He has had similar, though less severe, Past History.-He has had similar, though less severe,
attacks: the first was five years ago, and he has had four since, the last sevcn months ago, when he vomited bile and was in bed for three weeks.

Progress of the Case.-The pulse gradually quickened to 118 , the respirations to 36 , and the temperature varied between $97^{\circ}$ and $101^{\circ}$. Definite signs of a gaseous subphrenic abscess on the right side developed; it was noticed that the left loin was dull on percussion, and was oedematous. On March 8th $\mathrm{Mr}$. Dent opened the abdomen by an incision $3 \mathrm{in.} \mathrm{long} \mathrm{through} \mathrm{the} \mathrm{outer}$ side of the right rectus. On opening the peritoneum gas escaped, but there was not any pus. A large mass of thickened escaped there omenturas found extending to the left of the incision beneath the left rectus, and on separating this an abscess containing offensive pus was opened; a large drainage tube was inserted. A few drops of bile were noticed in the discharge; and, though the cause of the abscess was not found, it was thought to be probably a perforated duodenal ulcer. The wound drained freely after wound. The patient gradually sank and died on March 13th, 1910

The necropsy, performed by Dr. Trevor, showed that the gastro-colic omentum was attached by old adhesions to the anterior abdominal walls, from side to side, just below the costal margins. The under surface of the liver was adherent to the stomach, and there were adhesions around the gall bladder. There was a large subphrenic abscess on the left side of the upper abdomen, limited to the right by the left side of the falciform ligament of the liver, and occupying the dome of the stomach chamber. in short, the left anterior intraperitoneal sumarenamber; in short, the left anterior intraperitoneal subphrenic abscess as described by Barnard. A small track passing downwards and to the right under the free margin of the falciform ligament of the liver ran to a perforated duodenal ulcer the size of a shilling on the anterior surface just outside the pyloric ridge. A thin track of pus was found on the convexity of the liver to the right of the falciform ligament, but it did not spread out into an abscess; there were also one or two scattered collections of coagulated pus, the size of a threepenny piece, on the convexity of the right lobe of the liver, but the substance of the liver did not show any suppuration.: As these small purulent areas on the convexity of the right lobe of the liver were quite recent, it is probable that they had formed after the laparotomy.

It is noticeable that in this case the signs pointed to a right-sided gaseous subphrenic abscess, whereas the abscess was really on the left side. In explanation of this want of accord between the physical signs and the real condition, it is highly probable that there was free gas on the right side of the falciform ligament, though the abscess was on the left side. For at the operation air but no pus came out when the peritoneum was opened by the incision to the right of the right rectus muscle.

The main interest of the case is the occurrence of a leftsided subphrenic abscess due to a perforated duodenal ulcer. In this instance the history of past attacks of a similar, though less severe, nature and the presence of adhesions make it highly probable that there had been attacks of local peritonitis in connexion with the duodenal ulcer, and that the adhesions thus produced were responsible for the abscess being on the left side of the abdomen. Left-sided subphrenic abscesses due to perforated duodenal ulcer must be very rare, for the condition is not referred to in Moynihan's work on duodenal ulcer. ${ }^{8}$ From Box's remarks ${ }^{1}$ it appears that a left-sided intraperitoneal abscess might occur even in the absence of adhesions. He says : "The most posterior part of the general peritoneal cavity of the left subphrenic region is situate, when the body is supine, near the posterior border of the spleen, to the outer side of the upper end of the left kidney, just above the 